\title{
Acompanhamento dos dados de hanseníase na Bahia
}

\author{
Monitoring of leprosy data in Bahia \\ Seguimiento de los datos sobre lepra en Bahía
}

Recebido: 18/11/2021 | Revisado: 25/11/2021 | Aceito: 25/11/2021 | Publicado: 03/12/2021

\author{
Jonas Gomes Blanger \\ ORCID: https://orcid.org/0000-0002-1246-9741 \\ Faculdade Independente do Nordeste, Brasil \\ E-mail: jonaszzgb@gmail.com \\ Leia Alexandre Alves \\ ORCID: https://orcid.org/0000-0001-8931-2112 \\ Faculdade Independente do Nordeste, Brasil \\ E-mail: leia@ fainor.com.br \\ Vinícius Andrade Brito Santos \\ ORCID: https://orcid.org/0000-0001-5760-7311 \\ Faculdade Independente do Nordeste, Brasil \\ E-mail: vinnidrum@gmail.com
}

\begin{abstract}
Resumo
Este trabalho relata, descreve e discute os dados acerca da predominância da hanseníase na Bahia, fazendo também um comparativo com outra regiões brasileiras acerca desta infecção, uma vez que, trata sobre a ocorrência em todos os grupos etários, sendo que o Brasil é um dos países que mais contém casos de hanseníase no mundo, predominando em pessoas com idade acima de 60 anos, totalizando 24,7\% de todos os casos (World Health Organization, 2017). A partir dos dados fornecidos pela plataforma DATASUS sobre a frequência de diagnóstico de hanseníase na Bahia em homens e mulheres do período de 2017 a 2021 observa-se uma frequência total maior em homens entre os anos analisados, sendo que o total de casos em mulheres foi de 4947 casos e em homens foi um total de 6154 diagnósticos, representando assim, 1207 casos a mais em homens em relação às mulheres. Avaliando o gráfico 1, em que trata sobre a frequência de casos de hanseníase em todas as faixas etárias, observa-se a maior ocorrência de casos entre crianças e adolescentes de 10 a 15 anos, em todo o período dos dados coletados, sendo que, correspondeu com um total de $52,32 \%$ de todas as infecções notificadas na Bahia segundo os dados do DATASUS. Partindo para os jovens e adultos entre 20 a 59 anos, os contaminados corresponderam com um total de 33,36\%, sendo aí o segundo grupo que mais conteve infectados na região baiana, e por fim, os idosos de 60 a 80 anos ou mais, representando $14,32 \%$ dos hansênicos, sendo o grupo que menos sofreu infecções em todos os anos analisados desde 2017. A partir da análise do gráfico 2, observa-se os dados referentes ao total de número de casos de hanseníase na Bahia e do Nordeste, diagnosticados no período de 2017 à 2021, sendo que no primeiro ano analisado, os diagnósticos na Bahia totalizaram $24,09 \%$ do total da região nordestina, sendo o maior percentual dos períodos analisados, sendo que, o menor percentual foi em 2021, totalizando $11,82 \%$ dos casos do Nordeste. O perfil socioeconômico dos casos no estado da Bahia, considerada área endêmica e maior estado do Nordeste, é semelhante ao perfil do total de casos em todo o Brasil. O coeficiente de detecção em 2017, foi classificado como alto (14,5 casos por 100 mil habitantes), sendo que, $79,4 \%$ dos casos obtiveram cura.
\end{abstract}

Palavras-chave: Hanseníase; Bahia; Diagnóstico e infectados.

\begin{abstract}
This paper reports, describes and discusses the data on the prevalence of leprosy in Bahia, also making a comparison with other Brazilian regions about this infection, since it deals with the occurrence in all age groups, and Brazil is one of the countries that contains more cases of leprosy in the world, predominating in people over 60 years old, totaling $24.7 \%$ of all cases (World Health Organization, 2017). From the data provided by the DATASUS platform on the frequency of leprosy diagnosis in Bahia in men and women from the period 2017 to 2021 it is observed a higher total frequency in men among the years analyzed, being that the total number of cases in women was 4947 cases and in men was a total of 6154 diagnoses, thus representing 1207 more cases in men compared to women. Evaluating chart 1 , which deals with the frequency of leprosy cases in all age groups, it is observed the highest occurrence of cases among children and adolescents from 10 to 15 years old, in the entire period of the data collected, corresponding to a total of $52.32 \%$ of all infections reported in Bahia, according to data from DATASUS. Moving on to young people and adults aged 20 to 59 years, those infected corresponded with a total of $33.36 \%$, being the second group that contained the most infected in the Bahia region, and finally, the elderly aged 60 to 80 years or more, representing
\end{abstract}


$14.32 \%$ of leprosy patients, being the group that suffered the least infections in all the years analyzed since 2017. From the analysis of graph 2 , it is observed the data regarding the total number of leprosy cases in Bahia and the Northeast, diagnosed in the period from 2017 to 2021, and in the first year analyzed, the diagnoses in Bahia totaled $24.09 \%$ of the total in the northeastern region, being the highest percentage of the analyzed periods, and, the lowest percentage was in 2021, totaling $11.82 \%$ of the cases in the Northeast. The socioeconomic profile of cases in the state of Bahia, considered an endemic area and the largest state in the Northeast, is similar to the profile of the total number of cases throughout Brazil. The detection coefficient in 2017 was classified as high (14.5 cases per 100,000 inhabitants), and $79.4 \%$ of cases were cured.

Keywords: Leprosy; Bahia; Diagnosis and infected.

\section{Resumen}

Este trabajo informa, describe y discute los datos sobre la prevalencia de la lepra en Bahía, haciendo también una comparación con otras regiones brasileñas sobre esta infección, ya que se trata de la ocurrencia en todos los grupos de edad, y Brasil es uno de los países que contiene más casos de lepra en el mundo, predominando en personas mayores de 60 años, totalizando el 24,7\% de todos los casos (Organización Mundial de la Salud, 2017). A partir de los datos proporcionados por la plataforma DATASUS sobre la frecuencia de diagnósticos de lepra en Bahía en hombres y mujeres en el periodo de 2017 a 2021 se observa una mayor frecuencia total en hombres entre los años analizados, siendo que el número total de casos en mujeres fue de 4947 casos y en hombres fue un total de 6154 diagnósticos, representando así 1207 casos más en hombres en comparación con las mujeres. Evaluando el gráfico 1, que trata de la frecuencia de los casos de lepra en todos los grupos de edad, se observa la mayor ocurrencia de casos entre los niños y adolescentes de 10 a 15 años, a lo largo del período de los datos recogidos, correspondiendo a un total de 52,32\% de todas las infecciones notificadas en Bahía, según datos de DATASUS. Pasando a los jóvenes y adultos de 20 a 59 años, los infectados correspondieron con un total de 33,36\%, siendo allí el segundo grupo que contenía más infectados en la región de Bahía, y por último, los ancianos de 60 a 80 años o más, representando el 14,32\% de los enfermos de lepra, siendo el grupo que menos infecciones sufrió en todos los años analizados desde 2017. A partir del análisis del gráfico 2, se observan los datos referentes al número total de casos de lepra en Bahía y en el Nordeste, diagnosticados en el período de 2017 a 2021, y en el primer año analizado, los diagnósticos en Bahía totalizaron el 24,09\% del total de la región Nordeste, siendo el mayor porcentaje de los períodos analizados, y, el menor porcentaje fue en 2021, totalizando el $11,82 \%$ de los casos en el Nordeste. El perfil socioeconómico de los casos en el estado de Bahía, considerado un área endémica y el mayor estado del Nordeste, es similar al perfil del número total de casos en todo Brasil. El coeficiente de detección en 2017 se clasificó como alto (14,5 casos por cada 100.000 habitantes), y el 79,4\% de los casos se curaron.

Palabras clave: Lepra; Bahía; Diagnóstico e infectado.

\section{Introdução}

A hanseníase é uma doença infecciosa crônica causada por uma bactéria chamada Mycobacterium leprae (M. leprae), sendo que pode ocasionar o contágio, mas que é considerada uma doença de baixa infectividade, ou seja, a sua capacidade de transmissão é baixa. Em 2005 foi alcançada a meta de contaminação de menos de um caso para cada 10 mil habitantes pela maioria dos países, sendo que, nos dias atuais, a eliminação ou controle dos casos em diversos países continuam sendo um desafio (World Health Organization, 2017).

Através dos estudos acerca desta doença, nota-se que alguns fatores são responsáveis pelo descontrole epidemiológico da hanseníase em muitos países e regiões, como exemplo, o pouco conhecimento da população, o diagnóstico tardio, e a baixa estruturação da rede de atenção com o objetivo de promover assistência em saúde voltada para a hanseníase, que assim, contribui para o aumento de indivíduos contaminados (Monteiro et al. 2017).

O Brasil é um dos países que mais contém casos de hanseníase no mundo sendo que, em 2017 foram notificados 26.686 novos casos, em que, 24,7\% dos casos eram de pessoas com idade acima de 60 anos de idade ou mais (World Health Organization, 2017).

A hanseníase também é uma doença considerada por muitos como negligenciada, afetando e prevalecendo principalmente na população mais pobre, contribuindo também para a manutenção do quadro de desigualdade, já que pode representar um forte entrave ao desenvolvimento dos países (Gonçalves et al. 2017). 
Segundo o boletim epidemiológico da Organização Mundial de Saúde (OMS), a hanseníase está presente em todos os países do Continente Americano, sendo que, todos com exceção do Brasil, já descartaram a lepra como problema de saúde pública, que é caracterizada como menos de um caso a cada 10.000 habitantes em nível nacional (Gomes, et al. 2017).

A população está em processo de transição da estrutura etária, sendo que, poderão ser observadas mudanças notáveis nas faixas de idade entre 60 anos ou mais (Nobre, 2017). Em 2010, o Censo Demográfico brasileiro registrou 22.590.599 idosos portadores de hanseníase, cerca de $11,8 \%$ da população brasileira, sendo que, segundo as estimativas do Instituto Brasileiro de Geografia e Estatística (IBGE) para 2050 é de que o Brasil tenha cerca de 66.457 .570 idosos, correspondendo assim, 29,3\% da população brasileira (Departamento de Informática do SUS. http://tabnet.datasus.gov.br/cgi/sinannet/hanseniase/hans_indicadores.htm, acessado em 31/Out/2021), ou seja, ultrapassará da quantidade de pessoas com idade de 15 anos.

Vale ressaltar que o objetivo deste estudo é fazer um levantamento de dados sobre a hanseníase no estado da Bahia, com base nas variáveis de idade e gênero e traçar um comparativo com outros estados no Nordeste e demais regiões do país, com o intuito de contribuir de modo somatório e reunir as informações específicas dos casos de hanseníase no território baiano.

\section{Metodologia}

Trata-se de um estudo transversal ou ecológico com uso de dados abertos fornecidos pela plataforma DATASUS do período de 2017 a 2021, sendo que, após a coleta dos dados, foram feitas as observações necessárias, como exemplo, faixa etária de contaminação, sexo dos contaminados e comparação dos números de infecções entre os anos citados (Gordis, 2017). A pesquisa do estudo foi construída por todos os casos de infecção por hanseníase de pacientes da Bahia de todas as idades entre homens e mulheres. Por se tratar de um banco de dados de domínio público, não foi necessário a submissão do projeto ao Comitê de Ética em Pesquisa (Coutinho, 2014).

\section{Resultados e Discussão}

A partir dos dados fornecidos pela plataforma DATASUS sobre a frequência de diagnóstico de hanseníase na Bahia em homens e mulheres do período de 2017 a 2021 observa-se na tabela 1, uma frequência total maior em homens entre os anos analisados, sendo que o total de casos em mulheres foi de 4947 casos e em homens foi um total de 6154 diagnósticos, representando assim, 1207 casos a mais em homens em relação às mulheres. Vale ressaltar que em 2021, período mais próximo desde a observação e análise dos dados, foi o ano com os menores índices de diagnósticos em homens e mulheres, totalizando respectivamente, 663 e 511 casos, representando assim, uma diferença de 152 diagnósticos. De acordo com o documento publicado pela OMS, relativo à estratégia global para a hanseníase no período de 2016-2020, orienta para ações de saúde voltadas para crianças e mulheres (World Health Organization, 2016).

No entanto, a análise dos dados da Bahia revelam uma grande necessidade de desenvolvimento de estratégias voltadas para a população masculina, considerando uma tendência de crescimento da proporção de contatos examinados, que é menor quando o caso de hanseníase de referência é homem, mas vale lembrar que, a tendência de redução da proporção dos casos que têm alto índice de cura foi maior em homens, já a quantidade de abandonos e negligências no tratamento a tendência de redução que ocorre em ambos os sexos, foi maior entre as mulheres, e por fim, a iniciação de um novo tratamento por recidiva é maior no público masculino, havendo crescimento entre estes e clara redução na tendência apresentada para as mulheres. O Brasil foi o país que notificou o maior número de casos novos com entrada como recidiva no mundo, o que não necessariamente traduz a realidade do país e da Bahia. Tendo em vista que os critérios para o diagnóstico não são simples, é necessário implementar a vigilância de todos os casos para maior precisão, além da utilização adequada de exames complementares específicos (Martins, et al. 2014). 
A partir da Tabela 1 a seguir, pode-se avaliar e diferenciar a frequência de casos de hanseníase em homens e mulheres, destacando assim, a quantidade de diagnósticos de cada ano desde 2017 a 2021 em relação a cada gênero.

Tabela 1. Frequência de casos de hanseníase em homens e mulheres na Bahia.

\begin{tabular}{lcc}
\hline Ano do diagnóstico & Homens & Mulheres \\
\hline 2017 & 1545 & 1303 \\
2018 & 1498 & 1235 \\
2019 & 1447 & 1177 \\
2020 & 1001 & 721 \\
2021 & 663 & 511 \\
Total & $\mathbf{6 1 5 4}$ & $\mathbf{4 9 4 7}$ \\
\hline
\end{tabular}

Fonte: Ministério da Saúde/SVS - Sistema de Informação de Agravos de Notificação - Sinan Net. (2021).

Avaliando o Gráfico 1 a seguir, em que trata sobre a frequência de casos de hanseníase em todas as faixas etárias, observa-se a maior ocorrência de casos entre crianças e adolescentes de 10 a 15 anos, em todo o período dos dados coletados, sendo que, correspondeu com um total de 52,32\% de todas as infecções notificadas na Bahia segundo os dados do DATASUS. Partindo para os jovens e adultos entre 20 a 59 anos, os contaminados corresponderam com um total de $33,36 \%$, sendo o segundo grupo que mais conteve infectados na região baiana, e por fim, os idosos de 60 a 80 anos ou mais, representando $14,32 \%$ dos hansênicos, formando o grupo que menos sofreu infecções em todos os anos analisados desde 2017.

Gráfico 1. Frequência por ano de casos de hanseníase em todas as faixas etárias.

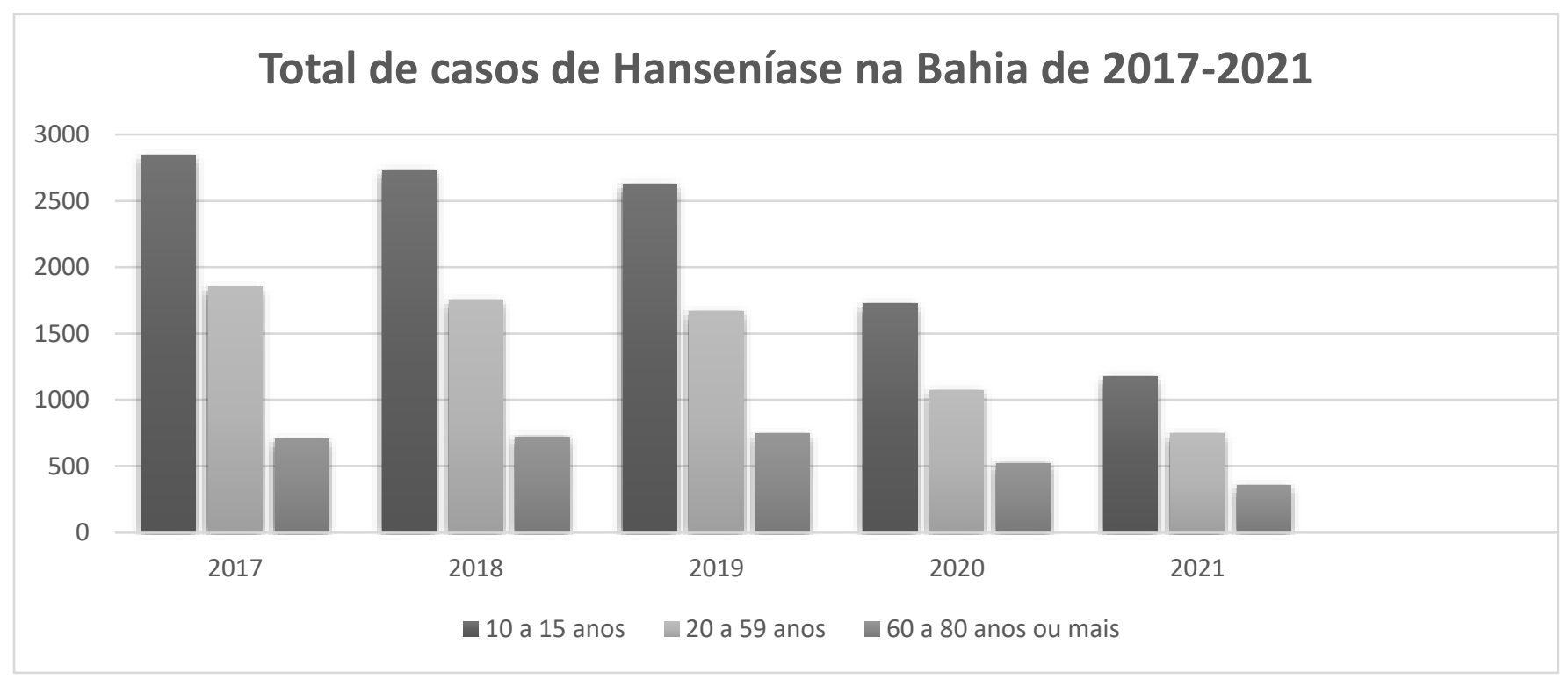

Fonte: Ministério da Saúde/SVS - Sistema de Informação de Agravos de Notificação - Sinan Net (2021). 
A partir dos dados expostos no gráfico, observa-se uma diferença de $10 \%$ com a média apresentada pela Organização Mundial de Saúde em 2017, em que afirmou que a quantidade de infectados com idade acima de 60 anos seria correspondente à $24 \%$ do total de casos no Brasil. Infelizmente, a hanseníase em crianças e adolescentes ainda é um indicador sensível do agravo e magnitude na população, demonstrando assim, eficácia limitada dos programas de saúde e permite a identificação dos fatores de risco (World Health Organization, 2016).

A presença de deformidades físicas e incapacidade motora em crianças e adolescentes podem sugerir permanência da transmissão da doença com o impacto negativo no rendimento escolar, nas atividades cotidianas, na interação com outras pessoas e no perfil psicológico (Freitas, et al. 2017). Há também alguns indicadores sobre a grande infecção entre as pessoas com idade de 15 anos ou menos, como exemplo, exposição precoce ao $M$. leprae e permanência da transmissibilidade da doença na comunidade (Carvalho, et al. 2015). Exposições e reexposições à bactéria transmissora de lepra durante relações extradomiciliares e domiciliares com casos da doença podem levar a contaminação, também é acrescentado nos estudos que a imaturidade imunológica das crianças pode favorecer à uma maior susceptibilidade a infecções (Rada, et al. 2012). Outros estudos demonstraram que crianças e adolescentes entre oito e 14 anos apresentam maiores possibilidades de desenvolver o agravo da hanseníase, sendo que, estes estudos também indicaram que a faixa etária mais acometida entre os menores de 15 anos estava entre 10 e 14 anos, sendo assim, pode se relacionar à resposta imunopatológica dos indivíduos, à fatores genéticos e ao longo período de incubacão da doença (Scheelbeek, et al. 2013).

Conhecer os fatores de riscos associados à lepra em contatos com pessoas menores de 15 anos contribui para ampliação de conhecimentos acerca desta doença com este público, reorientações das políticas públicas e das práticas dos serviços de saúde, favorecendo a fiscalização e aperfeiçoamento da vigilância da predominância e agravo nessa população específica, já que estudos demonstram que requer um cuidado maior. Também é necessária uma reeducação melhor e maior nas escolas e também para os indivíduos e familiares por parte dos profissionais de saúde, com a finalidade de contribuir para o conhecimento dos cuidados necessários para diminuir o risco de contaminação e também sobre a doença em si, contribuindo assim, para o exercício do autocuidado nessa população mais vulnerável (Scheelbeek, et al. 2013).

A partir da análise do gráfico 2, observa-se os dados referentes ao total de número de casos de hanseníase na Bahia e do Nordeste, diagnosticados no período de 2017 à 2021, sendo que no primeiro ano analisado, os diagnósticos na Bahia totalizaram $24,09 \%$ do total da região nordestina, sendo o maior percentual dos períodos analisados, sendo que, o menor percentual foi em 2021, totalizando 11,82\% dos casos do Nordeste. O perfil socioeconômico dos casos no estado da Bahia, considerada área endêmica e maior estado do Nordeste, é semelhante ao perfil do total de casos em todo o Brasil.

Gráfico 2. Comparativo do total de casos de hanseníase no Nordeste e na Bahia.

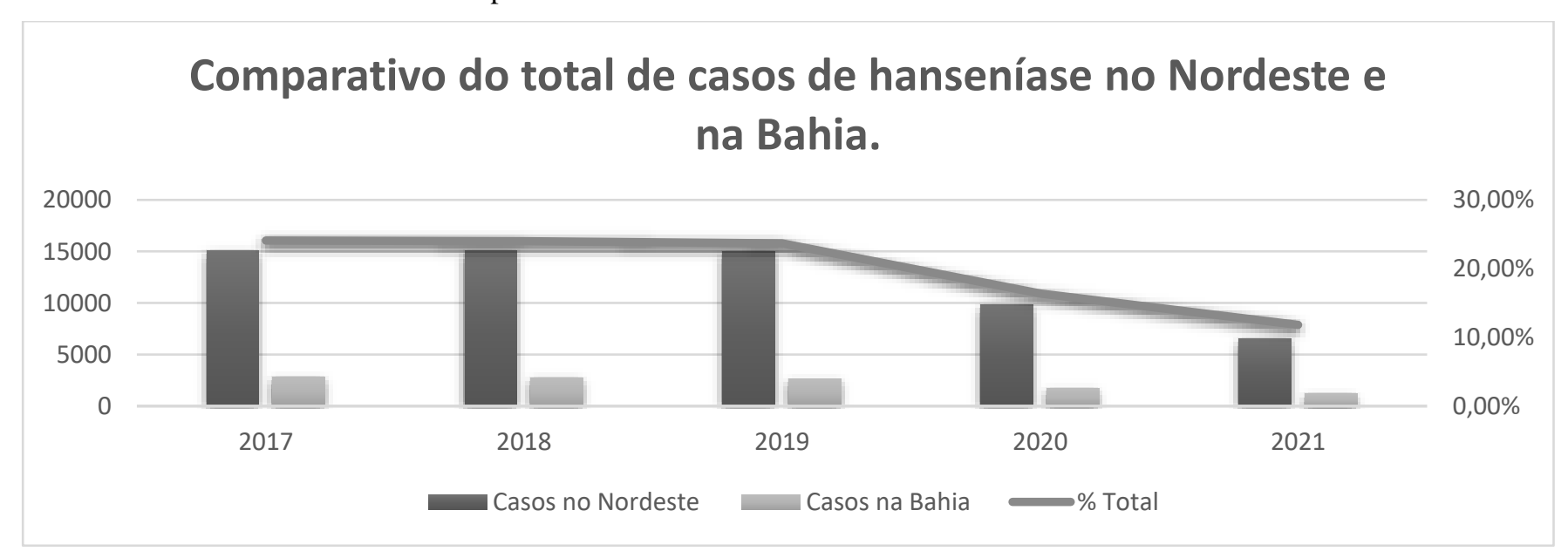

Fonte: Ministério da Saúde/SVS - Sistema de Informação de Agravos de Notificação - Sinan Net. (2021). 
O coeficiente de detecção em 2017, foi classificado como alto (14,5 casos por 100 mil habitantes), sendo que, 79,4\% dos casos obtiveram cura. Ainda, o maior número de casos novos da hanseníase nesse estado ocorre em pessoas do sexo masculino, cor parda, analfabeto ou ensino fundamental incompleto (Souza, et al. 2018). Sendo assim, os resultados indicam uma precariedade no controle de transmissão de hanseníase, quando se trata de atuação dos serviços de saúde, no que diz respeito ao desempenho das transmissões intradomiciliares, também vale ressaltar a importância do diagnóstico o mais cedo possível, diminuindo riscos de morte, de novas transmissões, aumentando assim, o controle de casos novos.

Avaliando alguns estudos que tratam acerca da contaminação por hanseníase devido ao índice de carência social (ICS), foi visto que, dos 417 municípios da Bahia, 15 (3,6\%) foram classificados como silenciosos, 27 (6,5\%) como de baixa endemicidade, $182(43,6 \%)$ como de média, 119 (28,5\%) como de alta, $35(8,4 \%)$ como muito alta endemicidade e $39(9,4 \%)$ como hiperendêmicos, sendo que os maiores coeficientes de contaminação estão concentrados na região norte-oeste do estado e na região sul e apenas 12 municípios apresentaram baixa carência social (Souza, 2020). O coeficiente de detecção de hanseníase nesse grupo foi bastante heterogêneo, variando entre 8,26/100 mil em Pojuca e 103,3/100 mil em Barreiras. Os municípios de Barreiras, Eunápolis, Teixeira de Freitas e Luís Eduardo Magalhães, sendo que foram classificados como cidades hiperendêmicas (Carlos, 2019). No outro extremo, tem-se os municípios com muito alta carência social, que representam 60,4\%. Desse total, 26 (10,3\%) foram classificados como de endemicidade baixa, sendo 11 deles totalmente silenciosos no período, e 18 (7,1\%) ccomo hiperendêmicos. Dos 10 municípios com maior ICS, quatro deles foram classificados como hiperendêmicos para a hanseníase. As regiões oeste e sul, prioritárias para hanseníase, apresentam menor carência social. Paralelamente, parte dos municípios do norte e centro-norte apresenta ICS alto e muito alto ao mesmo tempo que são super endêmicos (Carlos, 2019).

De acordo com os dados do Ministério da Saúde/SVS - Sistema de Informação de Agravos de Notificação - Sinan Net.2021, nos últimos quatro anos houveram 4947 casos de mulheres gestantes que foram contaminadas com a hanseníase, sendo que 253 destas mulheres abandonaram o tratamento. Segundo o ministério da saúde, uma mulher que tem hanseníase durante a gestação pode enfrentar diversos problemas, como exemplos, o bebê pode nascer prematuro e com baixo peso, além de poder apresentar sequelas, a mãe também pode sofrer algumas ocorrências não desejadas, como exemplo, pré-eclâmpsia e anemia (Brasil, 2020).

É recomendado que a mulher evite engravidar caso tenha acabado de ser diagnosticada com hanseníase ou que ainda esteja em tratamento, planejando assim, sua gravidez após estar definitivamente curada, mas caso isto aconteça, é necessário que procure o serviço de saúde para que o tratamento seja iniciado (Brasil, 2020).

\section{Conclusão}

Por meio deste estudo, ficou evidenciado as carências do estado da Bahia acerca dos cuidados para minimizar os casos de hanseníase baseado no panorama epidemiológico utilizado neste artigo para quantificar e avaliar os diagnósticos de lepra na Bahia envolvendo mulheres, homens, entre todas as idades a partir de 10 anos desde o ano de 2017 até 2021 em que ficou evidenciado uma quantidade maior de casos em homens em relação às mulheres e um predomínio em crianças e adolescentes entre 10 a 15 anos de idade. Como dito antes, isso ocorre provavelmente pelos seguintes indicadores: frequente exposições a pessoas contaminadas, exposição precoce ao M. leprae e permanência da transmissibilidade da doença na comunidade, além de que, a imaturidade imunológica das crianças pode favorecer à uma maior susceptibilidade a infecções.

Outro fator essencial e determinante para a frequência de contaminação é a carência social, em que, estudos demonstram que influencia diretamente no processo de adoecimento, a importância de definição de áreas prioritárias para intervenção de acordo com os aspectos epidemiológicos, principalmente em um estado onde condições básicas de saúde ainda são desiguais de acordo com as cidades, bairros e regiões, fazendo com que seja colocado em pauta a necessidade de 
particularizar cada região de acordo com suas necessidades e reforçando a importância de se estudar e avaliar a hanseníase, pois esta infecção ainda faz parte dos agravos históricos e ainda é muito negligenciada.

Por fim, é de suma importância a melhoria da qualidade da atenção e do acompanhamento até a conclusão do tratamento por parte do sistema público de saúde. De acordo com o Departamento de Vigilância das Doenças Transmissíveis, o estado da Bahia está inserido no parâmetro regular em relação à proporção de cura entre os casos de hanseníase do estado, segundo diretrizes adotadas pelo Ministério da Saúde, vale ressaltar sobre a importância de novos trabalhos e artigos que tratam sobre esta temática, para assim, atualizar os dados acerca da hanseníase, alertar, promover conhecimentos para a população em geral e também para os profissionais de saúde que estão à frente do controle desta doença, podendo conscientizar os leitores e auxiliar os profissionais no cuidado da hanseníase.

\section{Referências}

Brasil. Ministério da Saúde. Gabinete do Ministro. Gestantes e crianças também podem ter hanseníase. 2020.

Carvalho, A. P. M., Fabri, A. D. C. O. C., Oliveira, R. C., \& Lana, F. C. F. (2015). Factors associated with anti-phenolic glycolipid-I seropositivity among the household contacts of leprosy cases. BMC infectious diseases, 15(1), 1-8.

Coutinho, C. P. (2014). Metodologia de investigação em ciências sociais e humanas. Leya.

Departamento de Vigilância das Doenças Transmissíveis, Secretaria de Vigilância em Saúde, Ministério da Saúde. Diretrizes para vigilância, atenção e eliminação da hanseníase como problema de saúde pública. Manual técnico-operacional. Brasília: Ministério da Saúde; 2016.

Freitas, B. H. B. M. D., Cortela, D. D. C. B., \& Ferreira, S. M. B. (2017). Tendência da hanseníase em menores de 15 anos em Mato Grosso (Brasil), $2001-$ 2013. Revista de Saúde Pública, 51.

Gomes, F. C., Oliveira, T. C., Araújo, J. E. R., Félix, L. G., \& Araújo, K. M. F. A. (2014). Conhecimento do usuário da atenção primária à saúde acerca da hanseníase. Rev Enferm UFPE [Internet], 8(8), 3669-79.

Gonçalves, A., Mantellini, G. G., \& Padovani, C. R. (2010). Controle da hanseníase: perspectivas e aspectos epidemiológicos e operacionais. Revista do Instituto de Medicina Tropical de São Paulo, 52(6), 311-315.

Gordis, L. Epidemiologia. Thieme Revinter Publicações, 2017. p. 404

Hinds, P. S., Chaves, D. E., \& Cypess, S. M. (1992). Context as a source of meaning and understanding. Qualitative health research, 2(1), 61-74.

Martins, P. V., \& Iriart, J. A. B. (2014). Itinerários terapêuticos de pacientes com diagnóstico de hanseníase em Salvador, Bahia. Physis: Revista de Saúde Coletiva, 24, 273-289.

Monteiro, L. D., Alencar, C. H., Barbosa, J. C., Novaes, C. C. B. S., Silva, R. D. C. P. D., \& Heukelbach, J. (2014). Pós-alta de hanseníase: limitação de atividade e participação social em área hiperendêmica do Norte do Brasil. Revista Brasileira de Epidemiologia, 17, 91-104.

Nery, J. S., Pereira, S. M., Rasella, D., Penna, M. L. F., Aquino, R., Rodrigues, L. C., ... \& Penna, G. O. (2014). Effect of the Brazilian conditional cash transfer and primary health care programs on the new case detection rate of leprosy. PLoS neglected tropical diseases, 8(11), e3357.

Penna, M. L. F., Grossi, M. A. D. F., \& Penna, G. O. (2013). Perfil do país: Hanseníase no Brasil.

Rada, E., Duthie, M. S., Reed, S. G., Aranzazu, N., \& Convit, J. (2012). Serologic follow-up of IgG responses against recombinant mycobacterial proteins ML0405, ML2331 and LID-1 in a leprosy hyperendemic area in Venezuela. Memórias do Instituto Oswaldo Cruz, 107, 90-94.

Scheelbeek, P. F., Balagon, M. V., Orcullo, F. M., Maghanoy, A. A., Abellana, J., \& Saunderson, P. R. (2013). A retrospective study of the epidemiology of leprosy in Cebu: an eleven-year profile. PLoS neglected tropical diseases, 7(9), e2444.

Souza, C. D. F. D., Magalhães, M. A. F. M., \& Luna, C. F. (2020). Hanseníase e carência social: definição de áreas prioritárias em estado endêmico do Nordeste brasileiro. Revista brasileira de epidemiologia, 23, e200007.

Souza, E. A. D., Ferreira, A. F., Boigny, R. N., Alencar, C. H., Heukelbach, J., Martins-Melo, F. R., \& Ramos Jr, A. N. (2018). Hanseníase e gênero no Brasil: tendências em área endêmica da região Nordeste, 2001-2014. Revista de Saúde Pública, 52, 20.

World Health Organization. Global leprosy update, 2017: reducingthediseaseburdenduetoleprosy. WklyEpidemiolRec 2018; 93:445- 56.

World Health Organization. Global leprosy strategy 2016---2020. Accelerating towards a leprosy-free world. WHO http://www. searo.who.int/srilanka/areas/leprosy/global leprosy strategy 2016 2020.pdf.

World Health Organization. Global leprosy strategy 2016-2020: accelerating towards a leprosy-free world. http://apps.searo.who. int/PDS_DOCS/B5233.pdf 